\title{
Optical spectroscopy of BL Lac objects: New redshifts and mis-identified sources
}

\author{
N. Carangelo ${ }^{1,4}$, R. Falomo ${ }^{2}$, J. Kotilainen ${ }^{3}$, A. Treves ${ }^{4}$, and M.-H. Ulrich ${ }^{5}$ \\ 1 Università di Milano-Bicocca, Piazza della Scienza 3, 20126 Milano, Italy \\ 2 Osservatorio Astronomico di Padova, Vicolo dell'Osservatorio 5, 35122 Padova, Italy \\ e-mail: falomo@pd.astro.it \\ 3 Tuorla Observatory, University of Turku, Väisäläntie 20, 21500 Piikkiö, Finland \\ e-mail: jarkot@astro.utu.fi \\ 4 Università dell' Insubria, via Valleggio 11, 22100 Como, Italy \\ e-mail: treves@uninsubria.it \\ ${ }^{5}$ European Southern Observatory, ESO Headquarter Garching, Karl-Schwarzschild-Str. 2, 85748 Garching bei München, \\ Germany \\ e-mail: mhulrich@eso.org
}

Received 23 January 2003/ Accepted 12 June 2003

\begin{abstract}
We are carrying out a multi-purpose program of high signal to noise optical spectroscopy at medium resolution of 30 BL Lac objects. Here we report the detection of three new redshifts, and the discovery of three misclassified sources. The new redshifts refer to PKS 0754+100 $(z=0.266)$, H-1914-194 $(z=0.137)$ and 1ES0715-259, for which we derive a redshift of $z=0.465$ and also a "new" classification a Steep Spectrum Radio Quasar. In two cases (UM493 and 1620+103) stellar spectra indicate a wrong classification. The three sources with new redshift measurement show strong [OIII] emission in their spectra and their host galaxy properties are known. The central black hole masses derived using the $M_{\mathrm{BH}}-L_{\mathrm{bulge}}$ and the $M_{\mathrm{BH}}-\sigma([\mathrm{OIII}])$ relations are compared.
\end{abstract}

Key words. galaxies: BL Lacertae objects: general - galaxies: active - galaxies: quasars: general

\section{Introduction}

BL Lac objects are strong radio-loud sources that constitute a rather rare subclass of active galactic nuclei (AGN), distinguished by peculiar properties. In particular at variance with other classes of AGNs, BL Lacs are characterized by a lack of prominent emission lines, highly variable non-thermal continuum and strong, variable optical polarization. The quasi featureless optical spectra hinder the determination of the redshift (and therefore of the distance) for these sources. To date only for half of the possible population of BL Lacs ( $\sim 600$ objects, see Véron-Cetty \& Véron 2001) is the redshift known.

The redshift can be measured from the weak emission or absorption lines that are expected to come from the underlying galaxy, from the nucleus or, for very distant objects, from intervening material. The detection of these lines requires optical spectroscopy observations of appropriate signal-to-noise ratio and resolution and in particular depends on the brightness state of the object: during low state the weak non-thermal contribution of the nucleus allows the measurement of stellar lines of the host galaxy, while in the bright state their detection is

Send offprint requests to: N. Carangelo,

e-mail: nicoletta.carangelo@mib.infn.it more difficult, but the higher contrast renders more feasible the detection of intervening absorptions.

We are carrying out a systematic study of the optical spectra of $30 \mathrm{BL}$ Lacs observed during two campaigns at the $3.6 \mathrm{~m}$ ESO telescope. The study aims to (a) measure new redshifts, which is the subject of the present paper, (b) search for broad emission lines, (c) perform a statistical study of intervening absorption systems, and (d) measure the host galaxy velocity dispersion $\sigma$ (Treves et al. 2003; Falomo et al. 2003b). For point (a) we have concentrated on the brightest available targets taken from major multivalength surveys, for which there is no indication of a host galaxy. Preliminary results have been presented by Carangelo et al. (2002). The objects of the sample for which new redshifts or new classifications were found are reported in Table 1.

The structure of the paper is as follows: in Sect. 2 we describe the observations and data analysis, in Sect. 3 we report the results with comments on individual sources. Section 4 gives a summary and discussion of the results. In our analysis $H_{0}=50 \mathrm{~km} \mathrm{~s}^{-1} \mathrm{Mpc}^{-1}$ and $q_{0}=0$ were used to facilitate comparison with literature results (note that all objects have $z<0.5$ ). 
Table 1. The Journal of Observations: Col. (1) gives the name of the BL Lac Object, (2) and (3) the coordinates (2000), (4) the $V$ magnitude of the BL Lac (from the Véron-Cetty and Véron catalog), (5) the run of observations (RUN A: 24, 25, 26 July 2001; RUN B: 14, 15, 16 January 2002), (6) average signal-to-noise ratio $(S / N)$, (7) the exposure time.

\begin{tabular}{lllllcc}
\hline \hline Source & RA & DEC & $V$ & Run & $S / N$ & Texp (s) \\
$(1)$ & $(2)$ & $(3)$ & $(4)$ & $(5)$ & $(6)$ & $(7)$ \\
\hline 1ES 0715-259 & 071804.9 & -260811.0 & 18.0 & B & 70 & 1500 \\
PKS 0754+100 & 075706.6 & +095634.9 & 14.5 & B & 180 & 1200 \\
UM 493 & 122206.0 & -010638.0 & 16.4 & B & 60 & 600 \\
1620+103 & 162235.1 & +101314.0 & 18.0 & A & 70 & 1200 \\
H-1914-194 & 191745.1 & -192138.0 & 18.5 & A & 180 & 1200 \\
\hline
\end{tabular}

\section{Observations and data analysis}

Observations were gathered at La Silla (Chile) using the $3.6 \mathrm{~m}$ European Southern Observatory telescope equipped with the ESO Faint Object Spectrograph and Camera (EFOSC2) during two runs in July 2001 (run A) and in January 2002 (run B). We observed in dark conditions and the seeing was $0.6^{\prime \prime}-1.5^{\prime \prime}$. We obtained optical spectra at $\sim 12 \AA$ resolution $(F W H M)$ in the visible (4085-7520 $\mathrm{A}$ ) using a grism at $1.68 \AA$ /pixel dispersion combined with an $1.2^{\prime \prime}$ slit, centered on the object.

Standard data reduction was performed using different packages in $\operatorname{IRAF}^{1}$ in order to obtain 1-dimensional wavelength-calibrated extracted spectra. The spectra were also flux calibrated using several observations of spectrophotometric stars (Oke 1990) obtained on the same night.

For each object we secured 2-3 individual spectra and then combined them in order to improve the signal to noise ratio. This procedure helps to remove bad pixels and spurious features and to check the reliability of detected absorption and/or emission lines. A journal of the observations including the reached (average) $S / N$ is given in Table 1 .

\section{Results on individual sources}

1ES 0715-259 belongs to the Einstein Slew Survey (Elvis et al. 1992) and was classified as a BL Lac Object by Perlman et al. (1996). They assumed that a VLA radio source could be identified with the corresponding Slew Survey source if it fell within $80^{\prime \prime}$ of the X-ray position. However in this case they reported an offset in the radio-X-ray position of $>200^{\prime \prime}$. The VLA radio map of 1ES 0715-259 (Perlman et al. 1996) shows a clear FR II morphology.

The MMT spectrum obtained by Perlman et al. (1996) appeared featureless which motivated the BL Lac classification on the basis of the selection criteria of Stocke et al. (1991). The ratio $\log F_{x} / F_{r}=-5.2$ places $1 \mathrm{ES} 0715-259$ near the borderline between radio-selected and X-ray-selected BL Lac objects. There are no polarimetric data for this source.

${ }^{1}$ IRAF is distributed by the National Optical Astronomy Observatories, which are operated by the Association of the Universities for Research in Astronomy, Inc., under cooperative agreement with the National Science Foundation.
HST (Urry et al. 2000) clearly resolved 1ES0715-259 into a point source surrounded by a small, rather elongated host galaxy: both disk and de Vaucouleurs models can fit the data giving for the host galaxy an apparent magnitude of $R=21.1$ and $R=20.0$ respectively.

We took an optical spectrum of the counterpart of the radio source. This clearly shows strong emission lines (see Fig. 1) and the identification, as reported in Table 2, of the [OII], [NeIII], HeII blended with $\mathrm{H}_{\gamma}, \mathrm{H}_{\beta}$, [OIII] N1 and [OIII] N2 yields a redshift $\langle z\rangle=0.465 \pm 0.002$. On the basis of the line flux $F_{\text {line }}$ reported in Table 2, we derive line luminosities of $L_{\mathrm{H}_{\beta}} \sim 4.5 \times 10^{42} \mathrm{erg} \mathrm{s}^{-1}$ and $L_{[\mathrm{OIII]}} \sim$ $3 \times 10^{42} \mathrm{erg} \mathrm{s}^{-1}$ typical of radio loud quasar, while for BL Lacs typical $L_{\mathrm{H}_{\beta}} \sim 10^{40}-10^{41} \mathrm{erg} \mathrm{s}^{-1}$ (e.g. Sitko \& Junkkarinen 1985, their Table 2).

From our optical spectroscopy and the radio properties $\left(\alpha_{\mathrm{r}}=0.73\right.$, Perlman 2002, private communication) we propose that this source is not a BL Lac but a steep spectrum radio quasar (SSRQ). The mis-classification by Perlman et al. (1996) is likely due to the poor quality of their optical spectrum, although a dramatic variability of the source cannot be excluded.

PKS 0754+100 is a highly polarized and variable source that was discovered by Tapia et al. (1977) and classified as a blazar by Angel \& Stockman (1980). A first tentative redshift proposed by Persic \& Salucci $(1986, z=0.66)$ was questioned after the detection of the host galaxy by Abraham et al. (1991) and by Falomo (1996), who suggested $z \sim 0.3$ on the basis of the host galaxy properties (at $z=0.66$ the host would be extremely luminous: $M_{R} \simeq-25$ ).

Our spectrum (see Fig. 1) shows clearly two emission lines at $\lambda=4717.85 \AA$ and $\lambda=6339 \AA$ (see Table 2): identification of these features with [OII] (3727 $\AA$ ) and [OIII] (5007 $\mathrm{A})$ yields a redshift $\langle z\rangle=0.266 \pm 0.001$. This is consistent with the preliminary redshift proposed by Falomo \& Ulrich (2000, $z \simeq 0.28$ ). At $z=0.266$ the host galaxy luminosity becomes $M_{R}=-22.9$. We note that at the same redshift there is a companion galaxy, 13" NE, corresponding to a projected distance of $\sim 70 \mathrm{kpc}$ (Pesce et al. 1995).

UM 493, 1620+103 These objects are classified to date as BL Lacs in the main catalogs (e.g. Hewitt \& Burbidge 1993; Padovani \& Giommi 1995; Véron-Cetty \& Véron 2001). UM 493 was discovered in a thin-prism survey forextragalactic 

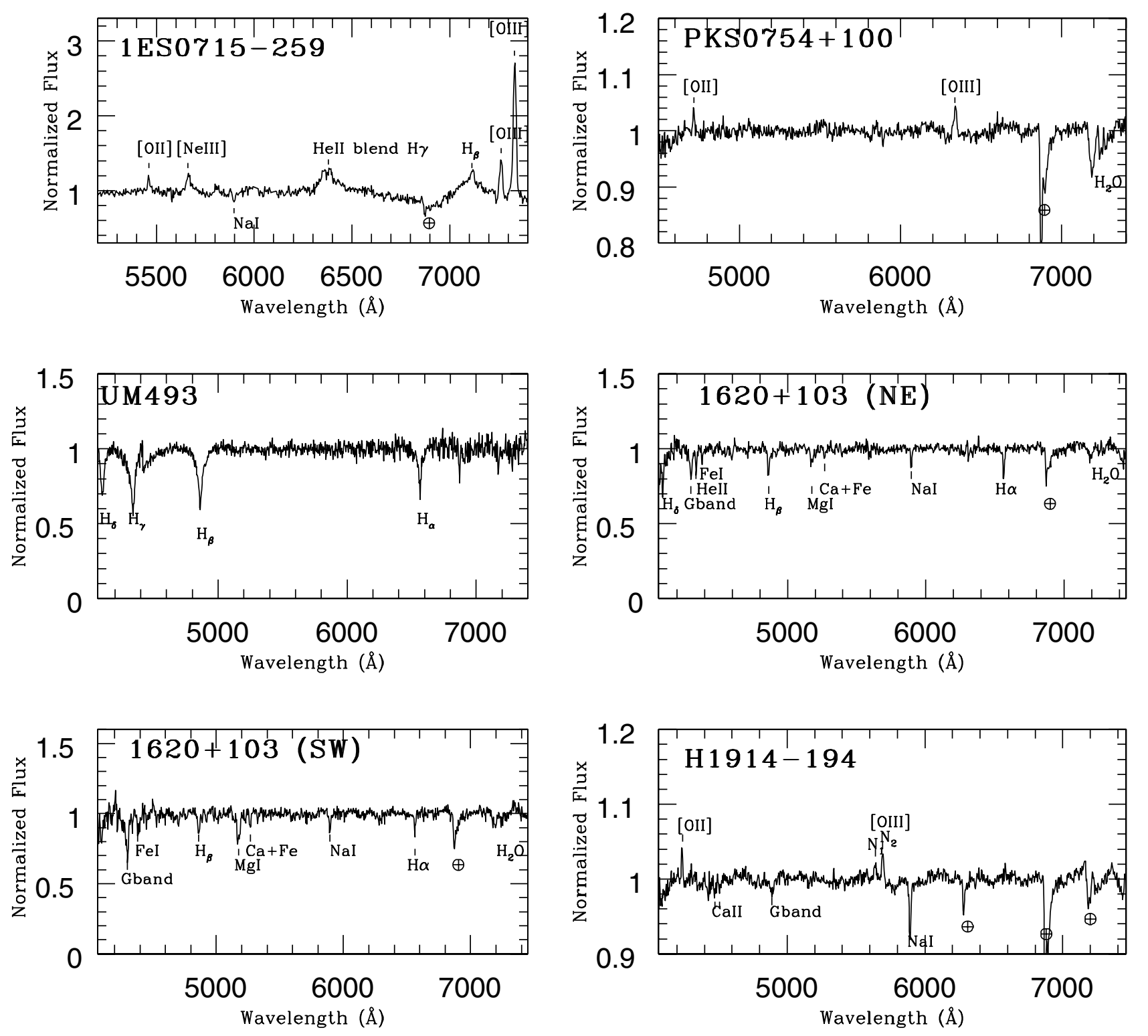

Fig. 1. Normalized optical spectra of the five sources: for each objects the detected emission and/or absorption lines are marked and identified. We note that in the case of 1ES0715-259 and H1914-194 we also observe an interstellar absorption line at $5892 \AA$ corresponding to NaI.

objects of the University of Michigan (MacAlpine \& Williams 1981). As reported in Fig. 1 the spectrum of this source shows absorption lines of the Balmer series rest-frame, clearly indicating that UM 493 is a star of spectral type A.

$1620+103$ is a radio source listed in the Molonglo catalogs MC2 and MC3 (Sutton et al. 1974). The radio position is given with a rms error in RA of $0.75^{\prime \prime}$ and of $10^{\prime \prime}$ in DEC (see Sutton et al. 1974). Hazard \& Murdoch (1977) proposed possible identification with two possible Blue Stellar Objects (BSOs) within $\pm \sim 23^{\prime \prime}$ of the radio position. Spectrophotometric observations for the sources, first by Baldwin et al. (1973), then by Smith et al. (1977) classified the SW object as a star and the NE object as a lineless object. Zotov \& Tapia (1979) revealed a polarization of $6 \%$ for the NE object, pointing to a BL Lac classification. McIlwrath \& Stannard (1980) suggested that the NE object is an example of a radio-quiet BL Lac.
We gathered spectra for both sources (NE and SW) of Hazard \& Murdoch (1977) (see Fig. 1): the NE object clearly shows the typical absorption line rest-frame of a galactic star and the SW object is confirmed to be a galactic star. We definitively conclude that the NE object is not a BL Lac but a star probably of spectral type $\mathrm{F}$ or intermediate A-F. The X-ray source detected by Della Ceca et al. (1990) (A-2 experiment aboard HEAO-1) coincides with the radio source but not with the star.

H-1914-194 is an X-ray-selected BL Lac (XBL) detected during the HEAO-1 Survey (Schwartz et al. 1989; Laurent-Muehleisen et al. 1993). This source was also detected in the radio during the Parkes-MIT-NRAO Survey (Griffith et al. 1994) and the Texas radio Survey (Douglas et al. 1996). Kollgaard et al. (1996) present a deep radio image for H-1914-194, which shows a bright core with a jetlike 
Table 2. Properties of emission and/or absorption lines: for each target the observed wavelengths of the detected emission (E) or absorption (A) lines, the identification, the redshift, the equivalent width $(E W)$ and for the emission lines the full width half maximum $(F W H M)$ corrected as described in the text and the flux observed are reported. In two case indicated as * in the table the $F W H M$ measured are very uncertain because the two emission lines blend.

\begin{tabular}{|c|c|c|c|c|c|c|c|}
\hline Source & $\lambda_{\mathrm{obs}}$ & $\mathrm{E} / \mathrm{A}$ & Ident. & (5) & $\begin{array}{l}E W \\
\AA \\
(6)\end{array}$ & $\begin{array}{l}F W H M \\
\mathrm{~km} \mathrm{~s}^{-1} \\
\text { (7) }\end{array}$ & $\begin{array}{l}\text { Flux } \\
\mathrm{erg} \mathrm{cm}^{-2} \mathrm{~s}^{-1} \\
\text { (8) }\end{array}$ \\
\hline \multirow[t]{7}{*}{ 1ES 0715-259 } & 5460.45 & $\mathrm{E}$ & [OII] & 0.465 & 1.9 & 360 & $1.44 \times 10^{-16}$ \\
\hline & 5662.21 & $\mathrm{E}$ & [NeIII] & 0.464 & 4.7 & 820 & $3.29 \times 10^{-16}$ \\
\hline & 6353.79 & $\mathrm{E}$ & HeII & 0.464 & 2.1 & $1010^{*}$ & $1.23 \times 10^{-16}$ \\
\hline & 6386.96 & $\mathrm{E}$ & $\mathrm{H}_{\gamma}$ & 0.471 & 4.1 & $1330^{*}$ & $2.46 \times 10^{-16}$ \\
\hline & 7116.19 & $\mathrm{E}$ & $\mathrm{H}_{\beta}$ & 0.464 & 49.4 & 5200 & $3.20 \times 10^{-15}$ \\
\hline & 7262.63 & $\mathrm{E}$ & [OIII] N1 & 0.465 & 11.9 & 630 & $7.48 \times 10^{-16}$ \\
\hline & 7333.04 & $\mathrm{E}$ & [OIII] N2 & 0.465 & 35.4 & 570 & $2.21 \times 10^{-15}$ \\
\hline \multirow[t]{2}{*}{ PKS 0754+100 } & 4717.85 & $\mathrm{E}$ & [OII] & 0.266 & 0.6 & 540 & $7.30 \times 10^{-16}$ \\
\hline & 6339.00 & $\mathrm{E}$ & [OIII] & 0.266 & 1.1 & 730 & $1.30 \times 10^{-15}$ \\
\hline \multirow[t]{6}{*}{ H-1914-194 } & 4240.14 & $\mathrm{E}$ & [OII] & 0.138 & 0.5 & 600 & $3 \times 10^{-15}$ \\
\hline & 4476.33 & A & CaII K & 0.138 & 0.3 & & \\
\hline & 4511.04 & $\mathrm{~A}$ & CaII H & 0.137 & 0.2 & & \\
\hline & 4890.92 & A & $G$ band & 0.136 & 0.3 & & \\
\hline & 5639.33 & $\mathrm{E}$ & [OIII] N1 & 0.137 & 0.3 & 800 & $3.80 \times 10^{-16}$ \\
\hline & 5693.55 & $\mathrm{E}$ & [OIII] N2 & 0.137 & 0.5 & 690 & $5.40 \times 10^{-16}$ \\
\hline
\end{tabular}

feature extended to the northwest toward a local brightening in a diffuse halo (see their Fig. 5a).

The optical counterpart is determined within $3^{\prime \prime}$ of the X-ray position: Kollgaard et al. (1996) give the optical position with an error of $\pm 0.3^{\prime \prime}$. HST imaging at high resolution (Scarpa et al. 2000; Urry et al. 2000) clearly resolved this source. The average radial profile of the host galaxy is consistent with both a de Vaucouleurs $\left(R_{\text {host }}=16.9\right)$ and a disk model $\left(R_{\text {host }}=17.9\right)$.

In our spectrum we clearly detect both nuclear emission lines and absorption lines from the host galaxy (see Fig. 1 and Table 2). Identification of these features with [OII], [OIII] N1 and N2, CaII H\&K and $G$ band yields a redshift of $\langle z\rangle=$ $0.137 \pm 0.001$.

\section{Discussion}

Our results are summarized in Fig. 1 and Table 2 where we report for each object the observed wavelengths of the detected emission (E) and/or absorption (A) lines, the identifications, the measured redshift, the equivalent width and for the emission lines the $F W H M$ and the flux observed. In particular we correct the measured $F W H M$ of the detected emission lines taking into account the instrumental resolution of $\sim 420 \mathrm{~km} \mathrm{~s}^{-1}$, that has been subtracted in quadrature from the measured widths. Note that this correction changes the line width by as much as $15-20 \%$.

We found for three sources that the BL Lac classification reported in the literature is wrong. In particular, 1ES 0715-259 is clearly a steep spectrum radio-loud quasar at a redshift of $z=0.465$ while UM493 and $1620+103$ are two stars. For the two confirmed BL Lac objects we measured new redshifts $z=$ 0.266 for PKS $0754+100$ and $z=0.137$ for H-1914-194.

From the measured $z$ we can derive the absolute magnitudes of the nuclei and of the host galaxies based on the apparent magnitudes measured with HST (Scarpa et al. 2000; Urry et al. 2000). Moreover we note that the three sources with determined redshifts show clear [OIII] emission in their spectra.

It is therefore possible to estimate the mass of the central black hole in two independent ways. The former is based on the $M_{\mathrm{BH}}-L_{\text {bulge }}$ relation (e.g. Kormendy \& Gebhardt 2001; Falomo et al. 2003a and references therein). In particular we have used the procedures described in detail by Bettoni et al. (2003). The results for the three objects under examination are reported in Table 3 . The uncertainties are $\sim 40 \%$. An alternative procedure is to start from the $\sigma$ ([OIII])- $\sigma^{*}$ (velocity dispersion) relation proposed by Nelson \& Whittle (1996), Nelson (2000), Shields et al. (2003), Boroson (2003), and to take advantage of the $M_{\mathrm{BH}}-\sigma$ relation (Ferrarese \& Merritt 2000; Gebhardt et al. 2000; Merritt \& Ferrarese 2001b). Even if our observations yield only a rough estimate of the [OIII] width, the derived $\sigma([\mathrm{OIII}])$ are in the range $\left(170-370 \mathrm{~km} \mathrm{~s}^{-1}\right)$, typical values of $\sigma^{*}$ measured in BL Lac objects (e.g. Barth et al. 2003; Falomo et al. 2002, 2003b; Treves et al. 2003). Mainly because of the large dispersion of the $M_{\mathrm{BH}^{-}}-\sigma([\mathrm{OIII}])$ relation, the resulting mass (Table 3 ) is more uncertain than with the previous 
Table 3. The black hole masses derived using the $M_{\mathrm{BH}}-L_{\text {bulge }}$ and $M_{\mathrm{BH}}-\sigma([\mathrm{OIII}])$ relations: Col. (1) gives the source name, (2) the redshift measured in this study, (3) the absolute $R$ host galaxy magnitude corrected for extinction, K-correction and evolution, (4) the $\mathrm{BH}$ mass derived using the $M_{\mathrm{BH}}-L_{\text {bulge }}$ relation, (5) the velocity dispersion $\sigma([\mathrm{OIII}])$ and (6) the $\mathrm{BH}$ mass derived from $M_{\mathrm{BH}^{-}} \sigma([\mathrm{OIII}]$ relation.

\begin{tabular}{cccccc}
\hline \hline Source & $z$ & $M_{R_{\text {host }}}$ & $\log \left(M_{\mathrm{BH}} / M_{\odot}\right)_{L_{\text {bulge }}}$ & $\sigma([\mathrm{OIII}])\left(\mathrm{km} \mathrm{s}^{1}\right)$ & $\log \left(M_{\mathrm{BH}} / M_{\odot}\right)_{\sigma([\mathrm{OIII}]}$ \\
$(1)$ & $(2)$ & $(3)$ & $(4)$ & $(5)$ & $(6)$ \\
\hline 1ES 0715-259 & 0.465 & -24.17 & 9.1 & 244 & 8.6 \\
PKS 0754+100 & 0.266 & -22.64 & 8.3 & 312 & 9.1 \\
H-1914-194 & 0.137 & -23.27 & 8.6 & 292 & 8.9 \\
\hline
\end{tabular}

technique, so that the results are not formally discordant. They indicate the uncertainties (Boroson 2003) and the potentiality of the method of determining $M_{\mathrm{BH}}$ for BL Lacs at large redshift, where the host galaxy is hard to detect.

Acknowledgements. We are grateful to Eric Perlman for comments. This research has made use of the NASA/IPAC Extragalactic Database (NED) which is operated by the Jet Propulsion Laboratory, California Institute of Technology, under contract with the National Aeronautics and Space Administration.

\section{References}

Abraham, R. G., McHardy, I. M., \& Crawford, C. S. 1991, MNRAS, 252, 482

Angel, J. R. P., \& Stockman, H. S. 1980, ARA\&A, 18, 321

Baldwin, J. A., Burbidge, E. M., Hazard, C., et al. 1973, ApJ, 185, 739

Barth, A., Ho, L. C., \& Sargent, W. L. W. 2003, ApJ, 588, 134

Bettoni, D., Falomo, R., Fasano, G., \& Govoni, F. 2003, A\&A, 399, 869

Boroson, T. A. 2003, ApJ, 585, 647

Carangelo, N., Falomo, R., Kotilainen, J., Treves, A., \& Ulrich, M. H. 2002, Conf. Proc., High Energy Blazar Astronomy, Finland, 17-21 June 2002, PASP Conf. Ser., to be published [astro-ph/0210016]

Della Ceca, R., Palumbo, G. G. C., Persic, M., et al. 1990, ApJS, 72, 471

Douglas, J. N., Bash, F. N., Bozyan, F. A. Torrence, G. W., \& Wolfe, C. 1996, AJ, 111, 1945

Elvis, M., Plummer, D., Schachter, J., \& Fabbiano, G. 1992, ApJS, 80, 257

Falomo, R. 1996, MNRAS, 283, 241

Falomo, R., \& Ulrich, M.-H. 2000, A\&A, 357, 91

Falomo, R., Kotilainen, J. K., \& Treves, A. 2002, ApJ, 569, L35

Falomo, R., Carangelo, N., \& Treves, A. 2003a, MNRAS, 343, 505

Falomo, R., Kotilainen, J., Carangelo, N., \& Treves, A. 2003b, ApJ, 595,624

Ferrarese, L., \& Merritt, D. 2000, ApJ, 539, L9

Gebhardt, K., Bender, R., Bower, G., et al. 2000, ApJ, 539, L13
Griffith, M. R., Wright, A. E., Burke, B. F., \& Ekers, R. D. 1994, ApJS, 90, 179

Hazard, C., \& Murdoch, H. S. 1977, AuJPA, 42, 1

Hewitt, A., \& Burbidge, G. 1993, ApJS, 87, 451

Kormendy, J., \& Gebhardt, K. 2001, AIP Conf. Proc., 586, 363

Kollgaard, R. I., Palma, C., Laurent-Muehleisen, S. A., \& Feigelson, E. D. 1996, ApJ, 465, 115

Laurent-Muehleisen, S. A., Kollgaard, R. I., Moellenbrock, G. A., \& Feigelson, E. D. 1993, AJ, 106, 875

MacAlpine, G. M., \& Williams, G. A. 1981, ApJS, 45, 113

McIlwrath, B. K., \& Stannard, D. 1980, MNRAS, 192, 79

Merritt, D., \& Ferrarese, L. 2001b, ApJ, 547, 140

Nelson, C. H., \& Whittle, M. 1996, ApJ, 465, 96

Nelson, C. H. 2000, ApJ, 544, L91

Oke, J. B. 1990, AJ, 99, 1621

Padovani, P., \& Giommi, P. 1995, MNRAS, 227, 1477

Perlman, E. S., Stocke, J. T., Schachter, J. F., et al. 1996, ApJS, 104, 251

Persic, M., \& Salucci, P. 1986, Struc. and Evol. of AGN, ed. Giuricin et al., 657

Pesce, J. E., Falomo, R., \& Treves, A. 1995, AJ, 110, 1554

Scarpa, R., Urry, C. M., Falomo, R., Pesce, J., \& Treves, A. 2000, ApJ, 532, 740

Schwartz, D. A., Brissenden, R. J. V., Tuohy, I. R., et al. 1989, in BL Lac objects, ed. L. Maraschi, T. Maccacaro, \& M.-H. Ulrich (Berlin: Springer), 209

Shields, G. A., Gebhardt, K., Salviander, S., et al. 2003, ApJ, 583, 124

Sitko, M. L., \& Junkkarinen, V. T. 1985, PASP, 97, 1158

Smith, H. E., Burbidge, E. M., Baldwin, J. A., et al. 1977, ApJ, 215, 427

Stocke, J. T., Morris, S. L., Gioia, I. M., et al. 1991, ApJS, 76, 813

Sutton, J. M., Davies, I. M., Little, A. G., \& Murdoch, H. S. 1974, AuJPA, 33, 1

Tapia, S., Craine, E. R., Gearhart, M. R., Pacht, E., \& Kraus, J. 1977, ApJ, 215, 71

Treves, A., Carangelo, N., Falomo, R., \& Kotilainen, J. 2003, ASP Conf. Ser., 290, 621

Urry, C. M., Scarpa, R., O’ Dowd, M., et al. 2000, ApJ, 532, 816

Véron-Cetty, M. P., \& Véron, P. 2001, A\&A, 374, 92

Zotov, N. V., \& Tapia, S. 1979, ApJ, 229, 5 